\title{
Construction of Nickel Oxide Nano-coral Structure on Microscope Slides for Total Self-Assembly Oriented Probe Immobilization and Signal Enhancement
}

Yu-Ling Hsieh, ${ }^{\dagger}$ Chien-Wei Chen, ${ }^{t, t}$ Wan-Hsuan Lin, ${ }^{\dagger}$ Bor-Ran Li ${ }^{*}, t, t, \$$

${ }^{\dagger}$ Institute of Biomedical Engineering, College of Electrical and Computer Engineering, National Chiao Tung University, 1001 University Road, Hsinchu 300, Taiwan

$\mp$ Department of Eectrical and Computer Engineering, College of Electrical and Computer Engineering, National Chiao Tung University, 1001 University Road, Hsinchu 300, Taiwan

$\S$ Center for Emergent Functional Matter Science, National Chiao Tung University, 1001 University Road, Hsinchu 300, Taiwan

Correspondence: liborran@g2.nctu.edu.tw

Tel: +886-3-5712-121\#54051; Fax: +886-3-5165-993 
Table S1. The detail of DNA sequence in this research.

\begin{tabular}{ccc}
\hline Name & Sequence from 5' to 3' & Length (bp) \\
\hline Target DNA & GCTGGAGCTCTGCAGCACACAGCAGTCGTATTAATTTCGC & 40 \\
Probe DNA & GCTGCAGAGCTCCAGC - Biotin & 16 \\
Reporter DNA & FITC - GCGAAATTAATACGAC & 16 \\
Single mutation & GCTGGAGGTCTGCAGCACACAGCAGTCGTATTAATTTCGC & 40 \\
Poly A & AAAAAAAAAAAAAAAAAAAAAAAAAAAAAAAAAAAAA & 40 \\
\hline
\end{tabular}



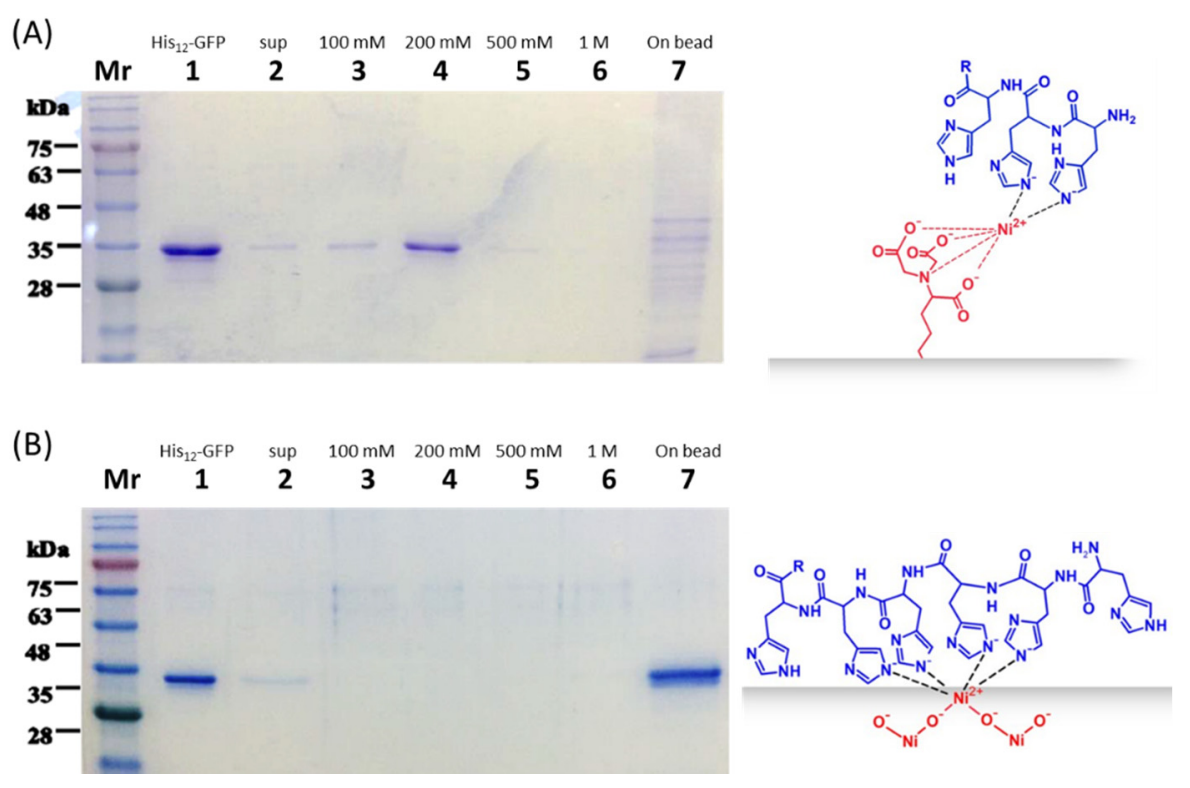

Figure S1. SDS-PAGE of eluting His12-GFP from (A) Ni-NTA Agarose and (B) NiO nanoparticle by different concentration of imidazole buffers and their relative binding mechanism.
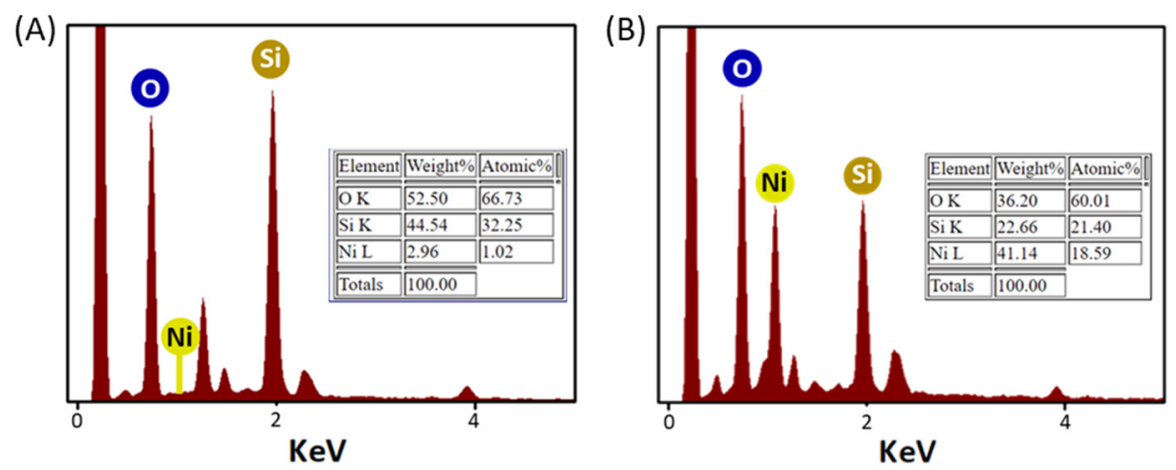

Figure S2. Comparison of element composition of (A) slide and (B) Ni-coated slide by EDS, HRSEM 

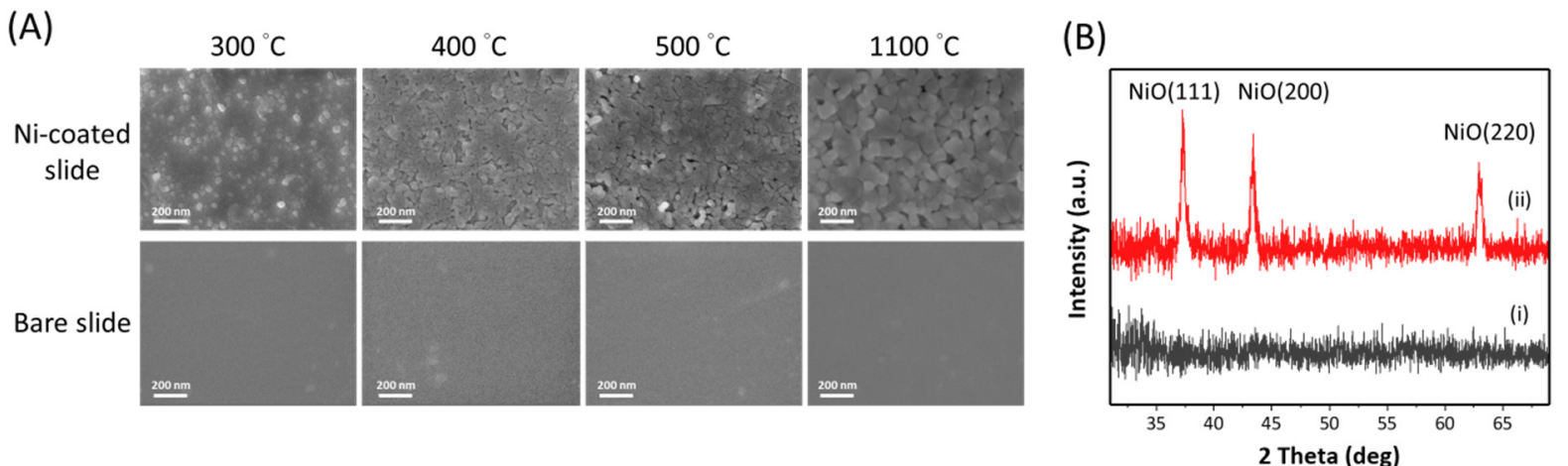

Figure S3. (A) SEM images of Ni-coated glass and glass under the same annealing conditions. (B) XRD patterns of Ni-coated glass and glass under the same annealing conditions (i) bare slide (ii) Ni-coated slide.
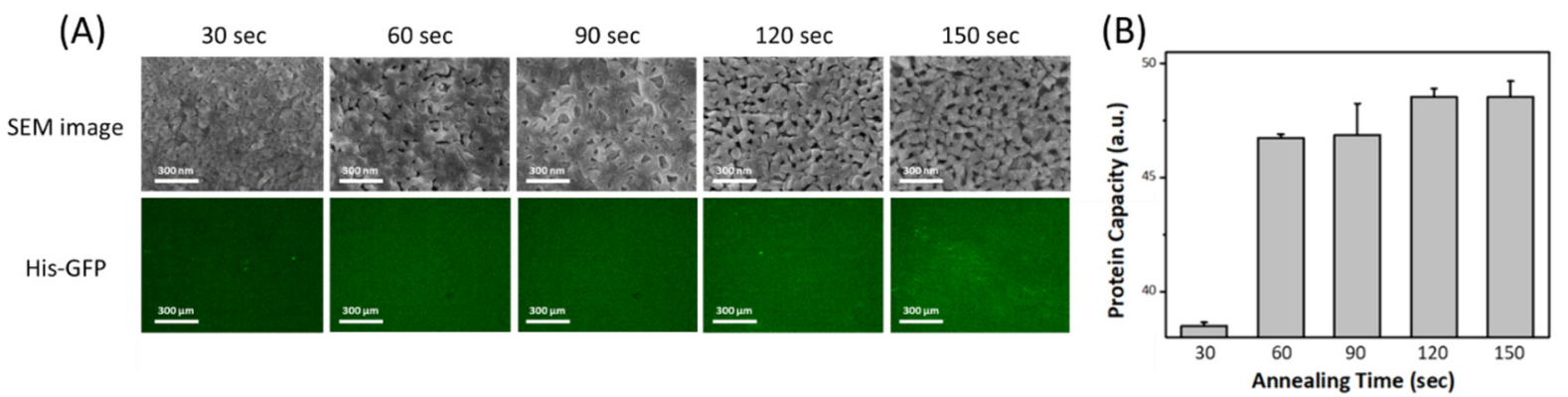

Figure S4. (A) Optimization of protein capacity after being annealed at different time. (B) Quantification of fluorescent intensity. 


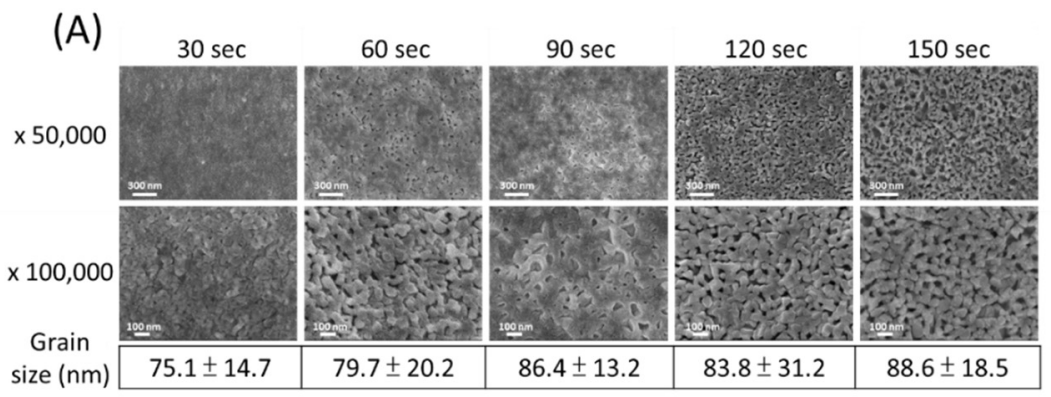

(B)

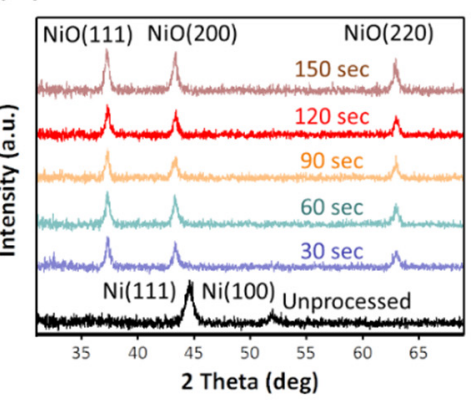

Figure S5. (A) SEM images of NiO thin film after applying different time of annealing (B) XRD spectrum of $\mathrm{NiO}$ thin film after applying different time of annealing.

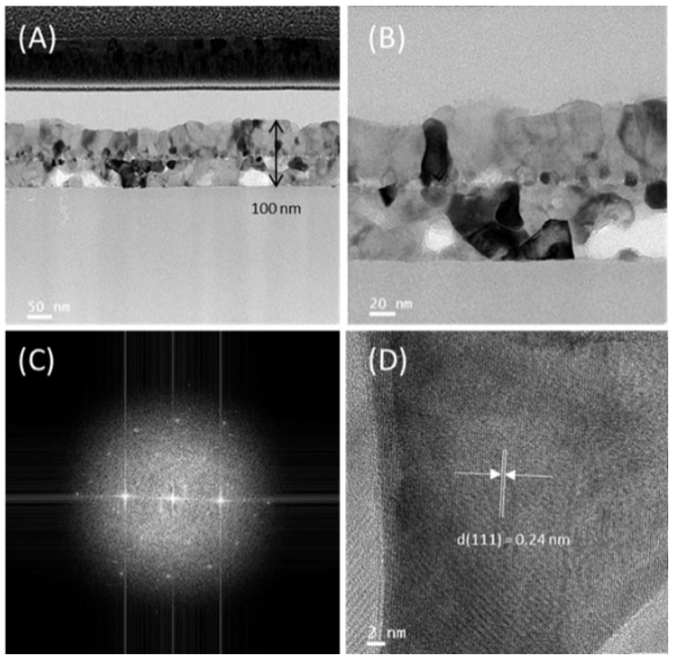

Figure S6. (A) Cross-sectional HRTEM image of nanostructured NiO thin film on the glass slide (B) zoomin image of Figure S6A (C) SAED image (D) lattice image of NiO crystal. 